Review Article

Cellular, Molecular and Developmental Genetics

\title{
Role of N6-methyl-adenosine modification in mammalian embryonic development
}

\author{
Chengshun $\mathrm{Li}^{1}$, Ziping Jiang ${ }^{2}$, Jindong $\mathrm{Hao}^{1}$, Da Liu ${ }^{3}$, Haobo $\mathrm{Hu}^{1}$, Yan $\mathrm{Gao}^{1}$ and Dongxu Wang ${ }^{1}$ \\ ${ }^{1}$ Jilin University, College of Animal Science, Laboratory Animal Center, Changchun, China. \\ ${ }^{2}$ The First Hospital of Jilin University, Department of hand surgery, Changchun, China. \\ ${ }^{3}$ Changchun University of Chinese Medicine, Department of Pharmacy, Changchun, China.
}

\begin{abstract}
N6-methyl-adenosine (m6A) methylation is one of the most common and abundant modifications of RNA molecules in eukaryotes. Although various biological roles of $\mathrm{m} 6 \mathrm{~A}$ methylation have been elucidated, its role in embryonic development is still unclear. In this review, we focused on the function and expression patterns of m6A-related genes in mammalian embryonic development and the role of $\mathrm{m} 6 \mathrm{~A}$ modification in the embryonic epigenetic reprogramming process. The modification of $\mathrm{m} 6 \mathrm{~A}$ is regulated by the combined activities of methyltransferases, demethylases, and m6A-binding proteins. m6A-related genes act synergistically to form a dynamic, reversible m6A pattern, which exists in several physiological processes in various stages of embryonic development. The lack of one of these enzymes affects embryonic m6A levels, leading to abnormal embryonic development and even death. Moreover, m6A is a positive regulator of reprogramming to pluripotency and can affect embryo reprogramming by affecting activation of the maternal-to-zygotic transition. In conclusion, $\mathrm{m} 6 \mathrm{~A}$ is involved in the regulation of gene expression during embryonic development and the metabolic processes of RNA and plays an important role in the epigenetic modification of embryos.
\end{abstract}

Keywords: m6A modification, embryo development, expression pattern, epigenetic reprogramming.

Received: August 04, 2020; Accepted: April 07, 2021.

\section{Introduction}

Modifications have been identified in different types of RNA, including mRNAs, tRNA, rRNA, and snoRNA, which are associated with various biological functions in mammals (Machnicka et al., 2013). RNA methylation has an important role in mRNA modifications, such as N6-methyl-adenosine (m6A) modification, which is the most common methylation modification (Niu et al., 2013; Frye et al., 2018). m6A was found to be highly enriched in 3' untranslated regions (UTR) or long internal exons, and 5' UTRs (Dominissini et al., 2012; Meyer et al., 2012; Batista et al., 2014; Ke et al., 2015; Zhang and Hamada, 2018). Moreover, m6A modifications are ubiquitous in prokaryotes, yeasts, and viruses (Chandola et al., 2015). Indeed, m6A is crucial for long non-coding RNAs, small nuclear RNAs, and ribosomal RNAs (Shimba et al., 1995; Piekna-Przybylska et al., 2008; Patil et al., 2016).

Most previous studies focused on gene and protein expression via DNA modification, such as DNA methylation and histone modifications (Toh et al., 2017; Xiang et al., 2019). However, there is limited research on RNA methylation modifications during embryonic development. RNA is widely involved in mammalian reproduction processes, such as the maturation of sperm and oocytes, embryonic development, and maintenance of the pluripotency of embryonic stem cells (Bettegowda and Smith, 2007; Sha et al., 2020). Previous reports showed that RNA was essential for embryonic and fetal

Send correspondence to Dongxu Wang. Jilin University, Laboratory Animal Center, College of Animal Science, 5333\#, Xi'an Road, Changchun 130062, China. E-mail: wang_dong_xu@jlu.edu.cn. development (Giraldez et al., 2005; Murchison et al., 2005; Melton et al., 2010; Stadler et al., 2010; Suh et al., 2010).

In this review, we analyzed the function and expression patterns of m6A-related genes in mammalian embryonic development. In addition, the role of m6A modification in the embryonic epigenetic reprogramming process was summarized.

\section{Effect of m6A methylation-related genes on embryonic development}

\section{Writers}

m6Amodification is regulated by several proteins including methyltransferase-like 3 (METTL3), methyltransferaselike 14 (METTL14), WTAP, and methyltransferase-like 16 (METTL16) referred to as "writers." They catalyze the m6A methylation of mRNA bases (Figure 1). METTL3 methylates the sequence motifRRACH $(\mathrm{R}=\mathrm{A}$ or $\mathrm{G} ; \mathrm{H}=\mathrm{A}, \mathrm{C}$ or $\mathrm{U})$ (Mendel et al., 2018). The heterodimer complex METTL3/ METTL14 catalyzes the m6A methylation modification of RNA in mammalian cells (Bokar et al., 1997; Wang Y et al., 2014; Meng et al., 2019). WTAP was the third protein identified in the m6A methyltransferase complex (Ping et al., 2014). Although WTAP has no methylation activity, it recruits METTL3/METTL14 heterodimer and promotes m6A methylation (Liu J et al., 2014). Previous studies reported that WTAP knockdown led to embryonic defects in mammals (Ping et al., 2014). The knockdown of METTL3 reduced m6A levels during mammalian embryonic development (Geula et al., 2015). The loss of METTL3 expression led to decreased m6A levels, which caused embryonic cells to remain in the 
naive state and consequently, resulted in embryonic lethality during the implantation phase (Table 1). METTL3 expression is important in normal meiosis, and the loss of expression of METTL3 led to defects in sperm meiosis (Geula et al., 2015; $\mathrm{Xu}$ et al., 2017). Indeed, METTL3 is required for development of the cerebellum, neurogenesis, and skin in mammals (Yoon et al., 2017; Wang C et al., 2018; Xi et al., 2020). In addition, METTL14 is indispensable for mouse post-implantation development (Meng et al., 2019). METTL16 catalyzes the methylation of the specific sequence UACAGAGAA in structured RNAs (Pendleton et al., 2017). In a previous study, the knockout of METTL16 led to the dysregulation of gene expression, which in turn, resulted in the failure of further development of implanted embryos (Mendel et al., 2018). These findings suggested that methyltransferase-mediated m6A modification is necessary for embryonic development.

\section{Erasers}

The demethylases involved in m6A modification are collectively known as "erasers" and include FTO and ALKBH5, belonging to the ALKB family (Kurowski et al., 2003; Gerken et al., 2007; Fu et al., 2010). FTO and ALKBH5

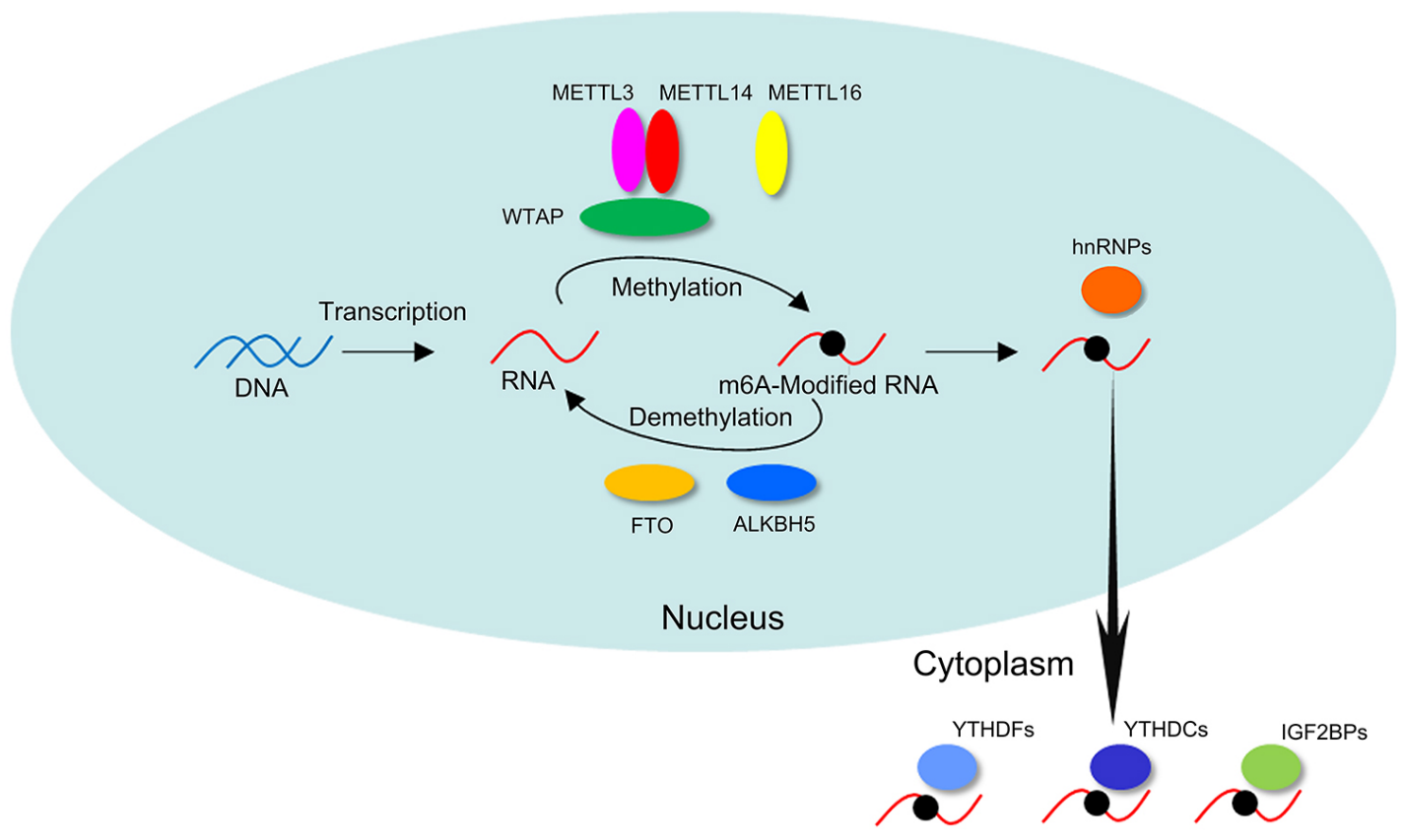

Figure 1 - The m6A modification process. METTL3, METTL14, WTAP, and METTL16 are methyltransferases, termed as "writers," involved in m6A modification in the nucleus. FTO and ALKBH5 are demethylases, termed as "erasers." YTHDFs, YTHDCs, and IGF2BPs are methylated reader proteins, referred to as "readers," located in the cytoplasm. Writers mediate m6A methylation, which is a post-transcriptional process in RNA. Erasers catalyze the demethylation of m6A, which reverses methylation. Readers recognize m6A modifications, which play a role in mRNA decay and translation.

Table $1-\mathrm{m}^{6} \mathrm{~A}$ methylation related to embryo development

\begin{tabular}{|c|c|c|c|c|}
\hline $\mathrm{m}^{6} \mathrm{~A}$-related protein & Function & Position & Knockout effect & References \\
\hline \multicolumn{5}{|c|}{ Methyltransferase (Writers) } \\
\hline \multirow[t]{2}{*}{ METTL3 } & RNA m ${ }^{6}$ A methylation & Nucleus & Embryo death & (Geula et al., 2015) \\
\hline & & & Stagnation of sperm meiosis & (Xu et al., 2017) \\
\hline \multirow[t]{2}{*}{ METTL14 } & RNA m ${ }^{6}$ A methylation & Nucleus & Embryo death & (Meng et al., 2019) \\
\hline & & & Stagnation of sperm meiosis & (Lin et al., 2017) \\
\hline METTL16 & RNA m ${ }^{6}$ A methylation & Nucleus & $\begin{array}{l}\text { Developmental arrest when } \\
\text { implantation }\end{array}$ & (Mendel et al., 2018) \\
\hline WTAP & $\begin{array}{l}\text { Regulatory subunit in } \mathrm{m}^{6} \mathrm{~A} \\
\text { methylation }\end{array}$ & Nucleus & Embryonic developmental defect & (Ping et al., 2014) \\
\hline \multicolumn{5}{|c|}{ Demethylase (Erasers) } \\
\hline FTO & Demethylation of $\mathrm{m}^{6} \mathrm{~A}$ RNA & Nucleus & Slow fetal growth & (Osborn et al., 2014) \\
\hline ALKBH5 & Demethylation of $\mathrm{m}^{6} \mathrm{~A}$ RNA & Nucleus & Sperm apoptosis & (Zheng et al., 2013) \\
\hline \multicolumn{5}{|c|}{ Binding proteins (Readers) } \\
\hline \multirow[t]{2}{*}{ YTHDC1 } & Identify m6A RNA & Nucleus & Embryo death & (Kasowitz et al., 2018) \\
\hline & & & Gametogenesis arrest & (Hsu et al., 2017) \\
\hline YTHDC & Identify m6A RNA & Nucleus & Adult fertility loss & (Zhao et al., 2017) \\
\hline YTHDF2 & Identify m6A RNA & Cytoplasm & Oocyte developmental defect & (Ivanova et al., 2017) \\
\hline IGF2BP1 & Identify m6A RNA & Cytoplasm & Embryo developmental defect & (Hao et al., 2020) \\
\hline
\end{tabular}


have been demonstrated to play a role in the reversible methylation of m6A in vitro and in vivo (Jia et al., 2011). FTO has been found to play a role in obesity and energy metabolism (Frayling et al., 2007; Fischer et al., 2009; Church et al., 2010; Yang et al., 2012). A recent study suggested that FTO is a demethylase involved in m6A modification (Jia et al., 2011). Reduction in the expression of FTO, which was detected in the nucleus and colocalized with nuclear speckles, induced weight loss or early death in mice (Fischer et al., 2009; Osborn et al., 2014). An increase in FTO expression levels, caused by GSK-3 deletion or inhibition, induced a decrease in m6A modification levels in mouse embryonic stem cells and reduced pluripotency (Faulds et al., 2018). ALKBH5 is the second mammalian m6A demethylase and ALKBH5-dependent m6A demethylation affects mRNA export (Zheng et al., 2013). Furthermore, ALKBH5 regulates spermatogenesis by reversing m6A methylation modification, resulting in the apoptosis of spermatocytes and impaired fertility in ALKBH5-deficient male mice. Based on these findings, it can be suggested that demethylases are involved in the dynamic process of m6A modification, which plays important roles in RNA export and metabolism, RNA processing factor assembly, and gene expression in mammalian embryonic development.

\section{Readers}

Methylated reading proteins, called "readers," are required to bind to $\mathrm{m} 6 \mathrm{~A}$ and perform the downstream functions of m6A modification. Readers include YTH domain proteins (YTHDF1, YTHDF2, YTHDF3, YTHDC1 and YTHDC2) and insulin-like growth factor 2 mRNA-binding proteins (IGF2BPs; including IGF2BP1, IGF2BP2 and IGF2BP3). YTHDF1, YTHDF2, and YTHDF3 are cytoplasmic readers. YTHDF1 and YTHDF3 work in concert to affect the translation of m6A RNA (Wang X et al., 2015; Shi et al., 2017) while YTHDF2 expedites mRNA decay (Wang X et al., 2014). The loss of YTHDF2 expression led to an imbalance in gene transcript regulation and affected oocyte development in mice (Ivanova et al., 2017). These findings indicated that YTHDF2 was a crucial factor in the acquisition of oocyte competence and during early zygotic development. YTHDC1 is essential for embryonic development in mice and localizes to the nucleus (Kasowitz et al., 2018). The inactivation of YTHDC1 leads to embryonic lethality. A previous study showed that although YTHDC2 knockout mice reached adulthood, both male and female mice were infertile (Hsu et al., 2017b). When readers bind to m6A-modified RNA, the targets of m6A-modified transcripts are activated during embryonic development. The loss of YTHDC2 expression caused abnormal m6A modification in mice (Wojtas et al., 2017). Thus, YTH domain proteins maintain mRNA stability and translation through m6A modification during embryonic development. IGF2BPs and YTHDF2 have a different function in the recognition and regulation of m6A RNA (Huang et al., 2018). Compared to YTH domain proteins, which mediate the mRNA decay pathway, IGF2BPs guard m6A-modified mRNA from decay. IGF2BPs, such as IGF2BP1, play roles in inhibiting mRNA degradation, increasing mRNA stability, and facilitating translation (Hao et al., 2020). Moreover, IGF2BPs as RNA-binding protein, play a potentially key role in embryonic development (Degrauwe et al., 2016). These data indicated that IGF2BPs are involved in various RNA biological processes in embryos at different developmental stages.

\section{Expression pattern of the genes responsible for regulating the $\mathrm{m} 6 \mathrm{~A}$ modification}

Increasing data have demonstrated that RNA modification is a dynamic process involved in the regulation of a variety of physiological processes (Motorin and Helm, 2011; Chan et al., 2012; Meyer and Jaffrey, 2014; Kirchner and Ignatova, 2015). Our previous data confirmed that m6A modification is a dynamic process that occurs during mouse embryonic development (Hao et al., 2019). This finding indicated that the methylation status of m6A was dynamically regulated by m6A-related genes during embryonic development (Faulds et al., 2018). The expression of m6A-related genes, such as METTL3, is important for the regulation of m6A modification during different embryonic stages (Kwon et al., 2019). In general, m6A levels increase slowly at the 2-cell, 4-cell, and 8-cell stages of mouse embryos. Notably, the factors associated with cellular pluripotency, such as OCT4, SOX2, and NANOG, were highly expressed in mouse embryonic cells. However, knocked down METTL3 and METTL14 reduced the expression of these pluripotency-related genes during embryonic development (Wang Y et al., 2014). m6A modification regulated gene expression, indicating that $\mathrm{m} 6 \mathrm{~A}$ modification determined RNA fate (i.e., transcription, splicing, or degradation) in the embryonic differentiation process (Young, 2011; Dunn et al., 2014; Yu et al., 2018). Compared to the 2-cell stage, the blastocyst stage exhibited increased m6A levels because of the active transcription of numerous genes. Thus, m6A levels show dynamic changes with increased expression during embryonic development.

The dynamic changes in m6A levels are attributed to methyltransferases (writers), demethylases (erasers), and methylation reader proteins (readers). These m6A-related genes are mainly involved in the production and removal of $\mathrm{m} 6 \mathrm{~A}$ RNA as well as the stabilization, translation, and degradation processes of m6A RNA. The network of methyltransferases, demethylases, and methylation reader proteins was analyzed using STRING bioinformatics software (Figure 2).METTL3/ METTL14 and WTAP as the core were connected to erasers and readers. Heterogeneous nuclear ribonucleoproteins (hnRNPs) such as hnRNP a2/b1 play important roles in m6A modification (Wu et al., 2018). HnRNP family members are mediators of m6A modification, which switch on RNA structural and regulated RNA-protein interactions (Liu N et al., 2015). Moreover, hnRNPu was found to be associated with IGF2BP1, which regulated m6A levels during mouse embryonic development (Weidensdorfer et al., 2009). In addition, hnRNPA2/B1 is crucial for gene transcripts and embryonic stem cell differentiation regulated by METTL3dependent m6A modification (Kwon et al., 2019). Furthermore, YTHDC1 and YTHDF1 influence mRNA splicing as m6A readers, whereas hnRNPc binds to m6A-modified RNAs to promote mRNA processing and alternative splicing (Hsu et al., 2017a). These data indicate that the loss of the expression of any m6A-related genes could lead to abnormal embryonic development in mammals. 


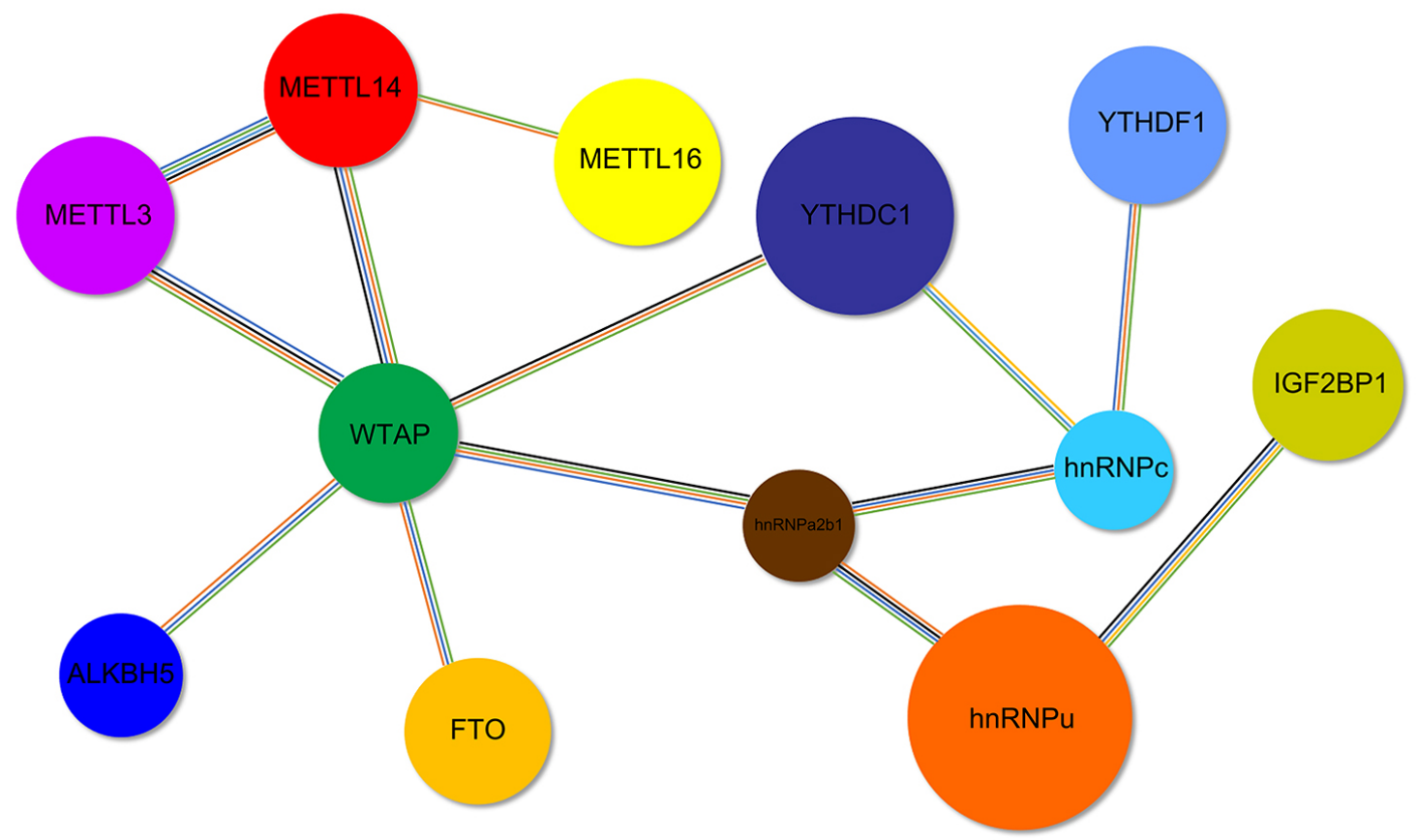

Figure 2 - Network of m6A-related genes. WTAP is a regulatory subunit required for the METTL3/METTL14 heterodimer complex. METTL16 is essential for RNA binding and methylation activity, and is associated with METTL14. FTO and ALKBH5 mediate RNA demethylation at the m6A modification site, causing unmethylated RNA to be bound by HuR protein, leading to constitutive expression. YTHDC1, YTHDF1, and IGF2BP1 recognize the methylation site and regulate mRNA degradation. hnRNPs regulate the expression of YTHDC1, YTHDF1, and IGF2BP1 in m6A modification.

\section{Role of $\mathrm{m} 6 \mathrm{~A}$ in epigenetic reprogramming of embryos}

DNA methylation and histone deacetylation are critical for epigenetic reprogramming during mammalian embryonic development. However, there are limited studies on m6A modification. m6A was found to be enriched in several functional groups, including chordate embryonic development, embryonic development, and gastrulation using m6A-sequencing (seq) analysis (Batista et al., 2014). This finding indicated that, like DNA methylation, dynamic and reversible m6A RNA modification could affect the epigenetic reprogramming of embryos (Figure 3). Increased m6A levels promoted the reprogramming of mouse embryonic fibroblasts into pluripotent stem cells, indicating that m6A modification is a positive regulator of the epigenetic reprogramming of embryos (Chen et al., 2015). The knockout of METTL3 leads to embryonic death, which might be related to decreases in m6A levels, causing abnormal reprogramming during early embryonic development in mice (Lin et al., 2017).

Maternal-to-zygotic transition (MZT) is a key process in which two differentiated gametes are reprogrammed during embryonic development. The clearance of maternal mRNAs and zygotic genome activation is realized during MZT (Zhao et al., 2017). Many maternal imprinted genes are eliminated through various transcriptional mechanisms to promote genome reprogramming in oocytes (Sui et al., 2020). m6A modification can affect embryonic reprogramming through transcriptional mechanisms involved in maternal clearance, which affect the stability of m6A RNA and promote the degradation of maternal mRNA. YTHDF2 plays an important role in this process by accelerating the degradation of mRNA. Loss of the expression of YTHDF2 did not clear maternal
mRNA and induced abnormal embryonic development (Zhao and $\mathrm{He}, 2017)$. m6A modification could influence embryonic reprogramming by regulating a variety of cytokines, including cell cycle regulators and pluripotency factors (Chen et al., 2015). Furthermore, m6A modification could promote the translation of key transcriptional activators of zygotes during MZT (Zhao et al., 2017). A recent study suggested that m6A modification controlled cell reprogramming to pluripotency (Alarcon et al., 2015). These findings demonstrated that m6A modification regulated epigenetic reprogramming during embryonic development.

m6A modification plays an important role in mouse embryo reprogramming by regulating RNA stability, degradation, translation, alternative splicing, and gene expression. m6A-related genes interact with regulatory factors to ensure the normal epigenetic reprogramming of embryos as a regulatory network.

In conclusion, the dynamic and reversible chemical m6A modification of RNA has shown effects on gene expression regulation and epigenetic reprogramming during embryonic development. The key factors affecting m6A levels are the three types of m6A-related enzymes: methyltransferases, demethylases, and methylation reader proteins. These proteins affect mRNA transcription, splicing, nuclear export, localization, and translation during embryonic development. The loss of expression of these proteins or their decreased activity can lead to the retardation of embryonic development and even death. Moreover, m6A modification plays an important role in the epigenetic reprogramming of embryonic development. Abnormal m6A levels can lead to defects in sperm or oocytes, fertility, and activation of the zygote genome. Thus, abnormal m6A modification leads to abnormal epigenetic reprogramming. 


\section{Spermatogenesis}

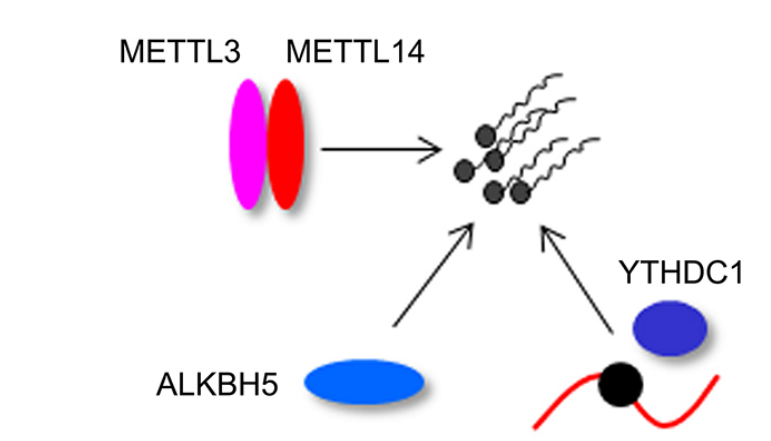

\section{Oocyte Development}

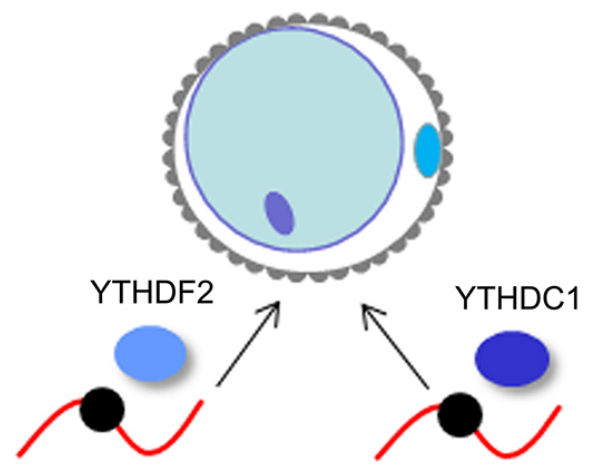

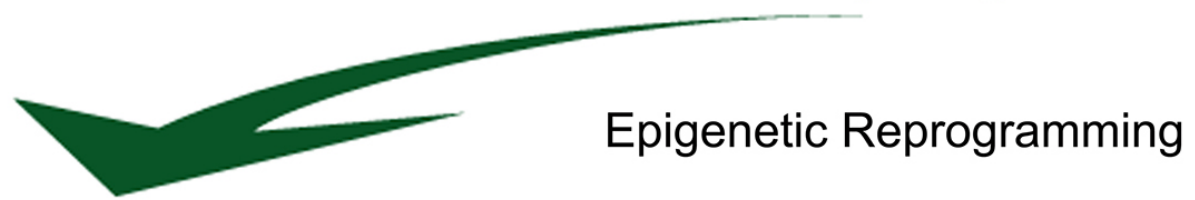

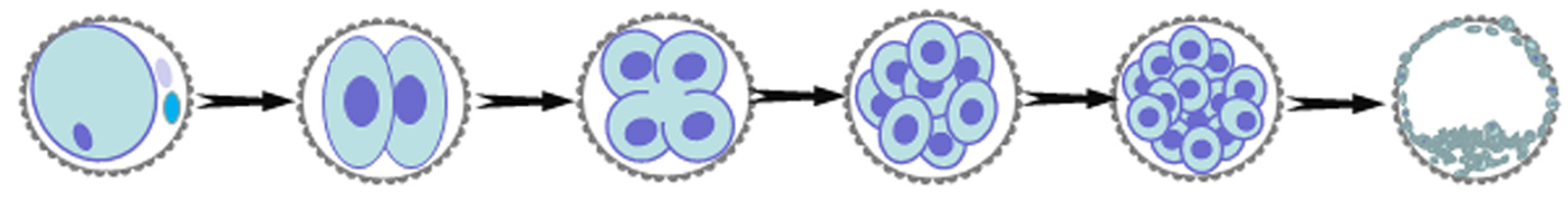

Dynamic and reversible

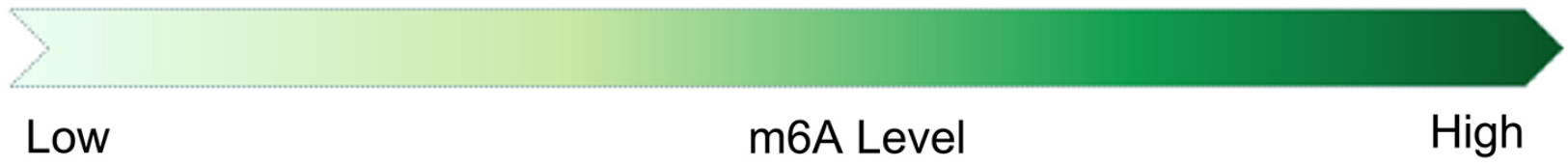

Figure 3 - Modification of m6A in embryonic epigenetic reprogramming. METTL3/METTL14, YTHDC1, and ALKBH5 play important roles in spermatogenesis. YTHDC1 and YTHDF2 are crucial in oocyte development. The dynamic and reversible m6A modification processes establish epigenetic reprogramming after fertilization. m6A levels increase during embryonic development.

\section{Acknowledgments}

This work was supported by the National Natural Science Foundation of China under Grant 81803680, the Fundamental Research Funds for the Central Universities under Grant 2019JCKT-70, the Jilin Education Department Program under Grant JJKH20200950KJ, the Jilin Province TCM science and technology project under Grant No. 2020041, and the Jilin Scientific and Technological Development Program under Grant 20190103071JH, 20170309005YY, and $20200504005 Y Y$.

\section{Conflict of Interest} interests.

The authors declare that they have no competing

\section{Author Contributions}

DW conceived the study, CL and JH wrote the manuscript, ZJ, DL, HH and YG prepared figures, all authors read and approved the final version.

\section{References}

Alarcón CR, Lee H, Goodarzi H, Halberg N and Tavazoie SF (2015) N6-methyladenosine marks primary microRNAs for processing. Nature 519:482-485.

Batista PJ, Molinie B, Wang J, Qu K, Zhang J, Li L, Bouley DM, Lujan E, Haddad B, Daneshvar K et al. (2014) m6 RNA modification controls cell fate transition in mammalian embryonic stem cells. Cell Stem Cell 15:707-719.

Bettegowda A and Smith GW (2007) Mechanisms of maternal mRNA regulation: implications for mammalian early embryonic development. Front Biosci 12:3713-3726.

Bokar JA, Shambaugh ME, Polayes D, Matera AG and Rottman FM (1997) Purification and cDNA cloning of the AdoMetbinding subunit of the human mRNA (N6-adenosine)methyltransferase. RNA 3:1233-1247.

Chan CT, Pang YL, Deng W, Babu IR, Dyavaiah, M, Begley TJ and Dedon PC (2012) Reprogramming of tRNA modifications controls the oxidative stress response by codon-biased translation of proteins. Nat Commun 3:937.

Chandola U, Das R and Panda B (2015) Role of the N6-methyladenosine RNA mark in gene regulation and its implications on development and disease. Brief Funct Genomics 14:169-179. 
Chen T, Hao YJ, Zhang Y, Li MM, Wang M, Han W, Wu Y, Lv Y, Hao $\mathrm{J}$ and Wang L et al.(2015) $\mathrm{m}^{6} \mathrm{~A}$ RNA methylation is regulated by microRNAs and promotes reprogramming to pluripotency. Cell Stem Cell 16:289-301.

Church C, Moir L, McMurray F, Girard C, Banks GT, Teboul L, Wells S, Bruning JC, Nolan PM and Ashcroft FM et al. (2010) Overexpression of Fto leads to increased food intake and results in obesity. Nat Genet 42:1086-1092.

Degrauwe N, Suva ML, Janiszewska M, Riggi N and Stamenkovic I (2016) IMPs: an RNA-binding protein family that provides a link between stem cell maintenance in normal development and cancer. Gene Dev 30:2459-2474.

Dominissini D, Moshitch-Moshkovitz S, Schwartz S, SalmonDivon M, Ungar L, Osenberg S, Cesarkas K, Jacob-Hirsch J, Amariglio N, Kupiec M et al. (2012) Topology of the human and mouse m6A RNA methylomes revealed by m6A-seq. Nature 485:201-206.

Dunn SJ, Martello G, Yordanov B, Emmott S and Smith AG (2014) Defining an essential transcription factor program for naive pluripotency. Science 344:1156-1160.

Faulds KJ, Egelston JN, Sedivy LJ, Mitchell MK, Garimella S, Kozlowski H, D'Alessandro A, Hansen KC, Balsbaugh JL and Phiel CJ (2018) Glycogen synthase kinase-3 (GSK-3) activity regulates mRNA methylation in mouse embryonic stem cells. J Biol Chem 293:10731-10743.

Fischer J, Koch L, Emmerling C, Vierkotten J, Peters T, Bruning JC and Ruther U (2009) Inactivation of the Fto gene protects from obesity. Nature 458:894-898.

Frayling TM, Timpson NJ, Weedon MN, Zeggini E, Freathy RM, Lindgren CM, Perry JR, Elliott KS, Lango H, Rayner NW et al. (2007) A common variant in the FTO gene is associated with body mass index and predisposes to childhood and adult obesity. Science 316:889-894.

Frye M, Harada BT, Behm M and He C (2018) RNA modifications modulate gene expression during development. Science 361:1346-1349.

Fu Y, Dai Q, Zhang W, Ren J, Pan T and He C (2010) The AlkB domain of mammalian ABH8 catalyzes hydroxylation of 5-methoxycarbonylmethyluridine at the wobble position of tRNA. Angew Chem Int Ed Engl 49:8885-8888.

Gerken T, Girard CA, Tung YC, Webby CJ, Saudek V, Hewitson KS, Yeo GS, McDonough MA, Cunliffe S, McNeill LA et al. (2007) The obesity-associated FTO gene encodes a 2-oxoglutaratedependent nucleic acid demethylase. Science 318:1469-1472.

Geula S, Moshitch-Moshkovitz S, Dominissini D, Mansour AA, Kol N, Salmon-Divon M, Hershkovitz V, Peer E, Mor N, Manor YS et al. (2015) Stem cells. m6A mRNA methylation facilitates resolution of naive pluripotency toward differentiation. Science 347:1002-1006.

Giraldez AJ, Cinalli RM, Glasner ME, Enright AJ, Thomson JM, Baskerville S, Hammond SM, Bartel DP and Schier AF (2005) MicroRNAs regulate brain morphogenesis in zebrafish. Science 308:833-838.

Hao J, Yu X, Gao W, Wei J, Qi M, Han L, Shi S, Lin C and Wang D (2019) The perturbed expression of m6A in parthenogenetic mouse embryos. Genet Mol Biol 42:666-670.

Hao J, Hu H, Jiang Z, Yu X, Li C, Chen L, Xia Y, Liu D and Wang D (2020) microRNA-670 modulates Igf2bp1 expression to regulate RNA methylation in parthenogenetic mouse embryonic development. Sci Rep 10:4782.

Hsu PJ, Shi H and He C (2017a) Epitranscriptomic influences on development and disease. Genome Biol 18:197.

Hsu PJ, Zhu YF, Ma HH, Guo YH, Shi XD, Liu YY, Qi MJ, Lu ZK, Shi HL, Wang JY et al. (2017b) Ythdc2 is an N-6-methyladenosine binding protein that regulates mammalian spermatogenesis. Cell Res 27:1115-1127.
Huang H, Weng H, Sun W, Qin X, Shi H, Wu H, Zhao BS, Mesquita A, Liu C, Yuan CL et al. (2018) Recognition of RNA N(6)methyladenosine by IGF2BP proteins enhances mRNA stability and translation. Nat Cell Biol 20:285-295.

Ivanova I, Much C, Di Giacomo M, Azzi C, Morgan M, Moreira PN, Monahan J, Carrieri C, Enright AJ and O'Carroll D (2017) The RNA $\mathrm{m}^{6} \mathrm{~A}$ reader YTHDF2 is essential for the post-transcriptional regulation of the maternal transcriptome and oocyte competence. Mol Cell 67:1059-1067.

Jia G, Fu Y, Zhao X, Dai Q, Zheng G, Yang Y, Yi C, Lindahl T, Pan T, Yang YG et al. (2011) N6-methyladenosine in nuclear RNA is a major substrate of the obesity-associated FTO. Nat Chem Biol 7:885-887.

Kasowitz SD, Ma J, Anderson SJ, Leu NA, Xu Y, Gregory BD, Schultz RM and Wang PJ (2018) Nuclear m6A reader YTHDC1 regulates alternative polyadenylation and splicing during mouse oocyte development. PLoS Genet 14:e1007412.

Ke S, Alemu EA, Mertens C, Gantman EC, Fak JJ, Mele A, Haripal B, Zucker-Scharff I, Moore MJ, Park CY et al. (2015) A majority of m6A residues are in the last exons, allowing the potential for 3' UTR regulation. Genes Dev 29:2037-2053.

Kirchner S and Ignatova Z (2015) Emerging roles of tRNA in adaptive translation, signalling dynamics and disease. Nat Rev Genet 16:98-112.

Kurowski MA, Bhagwat AS, Papaj G and Bujnicki JM (2003) Phylogenomic identification of five new human homologs of the DNA repair enzyme AlkB. BMC Genomics 4:48.

Kwon J, Jo YJ, Namgoong S and Kim NH (2019) Functional roles of hnRNPA2/B1 regulated by METTL3 in mammalian embryonic development. Sci Rep 9:8640.

Lin Z, Hsu PJ, Xing X, Fang J, Lu Z, Zou Q, Zhang KJ, Zhang X, Zhou Y, Zhang T et al. (2017) Mettl3-/Mettl14-mediated mRNA $\mathrm{N}(6)$-methyladenosine modulates murine spermatogenesis. Cell Res 27:1216-1230.

Liu J, Yue Y, Han D, Wang X, Fu Y, Zhang L, Jia G, Yu M, Lu Z, Deng X et al. (2014) A METTL3-METTL14 complex mediates mammalian nuclear RNA N6-adenosine methylation. Nat Chem Biol 10:93-95.

Liu N, Dai Q, Zheng G, He C, Parisien M and Pan T (2015) N(6)methyladenosine-dependent RNA structural switches regulate RNA-protein interactions. Nature 518:560-564.

Machnicka MA, Milanowska K, Osman Oglou O, Purta E, Kurkowska M, Olchowik A, Januszewski W, Kalinowski S, DuninHorkawicz S, Rother KM et al. (2013) MODOMICS: a database of RNA modification pathways--2013 update. Nucleic Acids Res 41:D262-267.

Melton C, Judson, RL and Blelloch R (2010) Opposing microRNA families regulate self-renewal in mouse embryonic stem cells. Nature 463:621-626.

Mendel M, Chen KM, Homolka D, Gos P, Pandey RR, McCarthy AA and Pillai RS (2018) Methylation of structured RNA by the m6A Writer METTL16 is essential for mouse embryonic development. Mol Cell 71:986-1000.e1011.

Meng TG, Lu X, Guo L, Hou GM, Ma XS, Li QN, Huang L, Fan $\mathrm{LH}, \mathrm{Zhao} \mathrm{ZH}, \mathrm{Ou} \mathrm{XH}$ et al. (2019) Mettl14 is required for mouse postimplantation development by facilitating epiblast maturation. FASEB J 33:1179-1187.

Meyer KD, Saletore Y, Zumbo P, Elemento O, Mason CE and Jaffrey SR (2012) Comprehensive analysis of mRNA methylation reveals enrichment in 3 ' UTRs and near stop codons. Cell 149:1635-1646.

Meyer KD and Jaffrey SR (2014) The dynamic epitranscriptome: N6-methyladenosine and gene expression control. Nat Rev Mol Cell Biol 15:313-326.

Motorin Y and Helm M (2011) RNA nucleotide methylation. Wiley Interdiscip Rev RNA 2:611-631. 
Murchison EP, Partridge JF, Tam OH, Cheloufi S and Hannon GJ (2005) Characterization of Dicer-deficient murine embryonic stem cells. Proc Natl Acad Sci U S A 102:12135-12140.

Niu Y, Zhao X, Wu YS, Li MM, Wang XJ and Yang YG (2013) N6-methyl-adenosine (m6A) in RNA: an old modification with a novel epigenetic function. Genomics Proteomics Bioinformatics 11:8-17.

Osborn DP, Roccasecca RM, McMurray F, Hernandez-Hernandez V, Mukherjee S, Barroso I, Stemple D, Cox R, Beales PL and Christou-Savina S (2014) Loss of FTO antagonises Wnt signaling and leads to developmental defects associated with ciliopathies. PloS One 9:e87662.

Patil DP, Chen CK, Pickering BF, Chow A, Jackson C, Guttman $M$ and Jaffrey SR (2016) m(6)A RNA methylation promotes XIST-mediated transcriptional repression. Nature 537:369-373.

Pendleton KE, Chen B, Liu K, Hunter OV, Xie Y, Tu BP and Conrad NK (2017) The U6 snRNA $m^{6} A$ methyltransferase METTL16 regulates SAM synthetase intron retention. Cell 169:824-835.e814.

Piekna-Przybylska D, Decatur WA and Fournier MJ (2008) The 3D rRNA modification maps database: with interactive tools for ribosome analysis. Nucleic Acids Res 36:D178-183.

Ping XL, Sun BF, Wang L, Xiao W, Yang X, Wang WJ, Adhikari S, Shi Y, Lv Y, Chen YS et al. (2014) Mammalian WTAP is a regulatory subunit of the RNA N6-methyladenosine methyltransferase. Cell Res 24:177-189.

Sha QQ, Zheng W, Wu YW, Li S, Guo L, Zhang S, Lin G, Ou XH and Fan HY (2020) Dynamics and clinical relevance of maternal mRNA clearance during the oocyte-to-embryo transition in humans. Nat Commun 11:4917.

Shi H, Wang X, Lu Z, Zhao BS, Ma H, Hsu PJ, Liu C and He C (2017) YTHDF3 facilitates translation and decay of N(6)methyladenosine-modified RNA. Cell Res 27:315-328.

Shimba S, Bokar JA, Rottman F and Reddy R (1995) Accurate and efficient N-6-adenosine methylation in spliceosomal U6 small nuclear RNA by HeLa cell extract in vitro. Nucleic Acids Res 23:2421-2426

Stadler B, Ivanovska I, Mehta K, Song S, Nelson A, Tan Y, Mathieu J, Darby C, Blau CA and Ware C et al. (2010) Characterization of microRNAs involved in embryonic stem cell states. Stem Cells Dev 19:935-950.

Suh N, Baehner L, Moltzahn F, Melton C, Shenoy A, Chen J and Blelloch R (2010) MicroRNA function is globally suppressed in mouse oocytes and early embryos. Curr Biol 20:271-277.

Sui X, Hu Y, Ren C, Cao Q, Zhou S, Cao Y, Li M, Shu W and Huo $\mathrm{R}$ (2020) METTL3-mediated $\mathrm{m}^{6} \mathrm{~A}$ is required for murine oocyte maturation and maternal-to-zygotic transition. Cell Cycle 19:391-404.

Toh TB, Lim JJ and Chow EK (2017) Epigenetics in cancer stem cells. Mol Cancer 16:29.

Wang CX, Cui GS, Liu XY, Xu K, Wang M, Zhang XX Jiang LY, Li A, Yang Y, Lai WY et al. (2018) METTL3-mediated m(6) A modification is required for cerebellar development. PLoS Biol 16:e2004880.

Wang X, Lu Z, Gomez A, Hon GC, Yue Y, Han D, Fu Y, Parisien M, Dai Q, Jia G et al. (2014) N6-methyladenosine-dependent regulation of messenger RNA stability. Nature 505:117-120.

Wang X, Zhao BS, Roundtree IA, Lu Z, Han D, Ma H, Weng X, Chen $\mathrm{K}$, Shi H and He C (2015) N(6)-methyladenosine modulates messenger RNA translation efficiency. Cell 161:1388-1399.
Wang Y, Li Y, Toth JI, Petroski MD, Zhang Z and Zhao JC (2014) N6-methyladenosine modification destabilizes developmental regulators in embryonic stem cells. Nat Cell Biol 16:191-198.

Weidensdorfer D, Stohr N, Baude A, Lederer M, Kohn M, Schierhorn A, Buchmeier S, Wahle E and Huttelmaier S (2009) Control of c-myc mRNA stability by IGF2BP1-associated cytoplasmic RNPs. RNA 15:104-115.

Wojtas MN, Pandey RR, Mendel M, Homolka D, Sachidanandam $\mathrm{R}$ and Pillai RS (2017) Regulation of $\mathrm{m}^{6} \mathrm{~A}$ transcripts by the 3 ' $\rightarrow$ ' ' RNA Helicase YTHDC2 is essential for a successful meiotic program in the mammalian germline. Mol Cell 68:374387.e312.

Wu B, Su S, Patil DP, Liu H, Gan J, Jaffrey SR and Ma J (2018) Molecular basis for the specific and multivariant recognitions of RNA substrates by human hnRNP A2/B1. Nat Commun 9:420.

Xi L, Carroll T, Matos I, Luo JD, Polak L, Pasolli HA, Jaffrey SR and Fuchs E (2020) m6A RNA methylation impacts fate choices during skin morphogenesis. Elife 9:e56980.

Xiang Y, Guo Z, Zhu P, Chen J and Huang Y (2019) Traditional Chinese medicine as a cancer treatment: Modern perspectives of ancient but advanced science. Cancer Med 8:1958-1975.

Xu K, Yang Y, Feng GH, Sun BF, Chen JQ, Li YF, Chen YS, Zhang XX, Wang CX, Jiang LY et al. (2017) Mettl3-mediated m(6)A regulates spermatogonial differentiation and meiosis initiation. Cell Res 27:1100-1114.

Yang J, Loos RJ, Powell JE, Medland SE, Speliotes EK, Chasman DI, Rose LM, Thorleifsson G, Steinthorsdottir V and Magi $\mathrm{R}$ et al. (2012) FTO genotype is associated with phenotypic variability of body mass index. Nature 490:267-272.

Yoon KJ, Ringeling FR, Vissers C, Jacob F, Pokrass M, Jimenez-Cyrus D, Su Y, Kim NS, Zhu Y, Zheng L et al. (2017) Temporal control of mammalian cortical neurogenesis by m(6)A methylation. Cell 171:877-889.e817.

Young RA (2011) Control of the embryonic stem cell state. Cell 144:940-954

Yu J, Chen M, Huang H, Zhu J, Song H, Zhu J, Park J and Ji SJ (2018) Dynamic m6A modification regulates local translation of mRNA in axons. Nucleic Acids Res 46:1412-1423.

Zhang Y and Hamada M (2018) DeepM6ASeq: prediction and characterization of m6A-containing sequences using deep learning. BMC Bioinformatics 19:524.

Zhao BS and He C (2017) "Gamete On" for $\mathrm{m}^{6} \mathrm{~A}$ : YTHDF2 exerts essential functions in female fertility. Mol Cell 67:903-905.

Zhao BS, Wang X, Beadell AV, Lu Z, Shi H, Kuuspalu A, Ho RK and $\mathrm{He} \mathrm{C}(2017) \mathrm{m}^{6} \mathrm{~A}$-dependent maternal mRNA clearance facilitates zebrafish maternal-to-zygotic transition. Nature 542:475-478.

Zheng G, Dahl JA, Niu Y, Fedorcsak P, Huang CM, Li CJ, Vagbo CB, Shi Y, Wang WL and Song SH et al. (2013) ALKBH5 is a mammalian RNA demethylase that impacts RNA metabolism and mouse fertility. Mol Cell 49:18-29.

Associate Editor: Igor Schneider

License information: This is an open-access article distributed under the terms of the Creative Commons Attribution License (type CC-BY), which permits unrestricted use distribution and reproduction in any medium, provided the original article is properly cited. 